DOI: $10.4274 /$ tpa.967

\title{
Evaluation of gene SCNN1A responsible for the synthesis of alpha subunit of epithelial sodium channel in transient tachypnea of newborn
}

\author{
Osman Öztekin1, Mahmut Akyol2, Salin Kalay1', Gönül Tezel1, Mustafa Akçakuş1, Nihal Oygür1 \\ ${ }^{1}$ Akdeniz University Medical Faculty, Department of Pediatrics, Division of Neonatology, Antalya, Turkey \\ 2Akdeniz University Medical Faculty, Department of Medical Biology and Genetics, Antalya, Turkey
}

\begin{abstract}
Summary
Aim: Epithelial sodium channels play an important role in the regulation of movement of sodium and absorption of alveolar fluid during prenatal period. Transient tacyhpnea of the newborn is a common respiratory problem in term or near-term infants. A lack of maturation of epithelial sodium channels is considered in its etiology. However, the role of genetic factors is unclear. The purpose of this study is to determine the role of gene SCNN1A which is responsible for synthesis of epithelial sodium channel alpha unit which is known to be effective in the mechanism of the disorder.

Material and Method: Newborn infants $>37$ weeks of gestation with a diagnosis of transient tacyhpnea of the newborn followed up at Neonatal Intensive Care Unit of Akdeniz University Medical Faculty between July 2010 - January 2012 and a control group consisting of healthy infants at the same gestational age were included in the study. $2 \mathrm{cc}$ of blood with EDTA was obtained from both groups in the first five days of their life and blood samples were kept at $-80^{\circ}$ degree. DNA isolation was fulfilled in the Department of Medical Biology. The gene was displayed using BigDye Terminator with the method of Sanger sequencing. The study was approved by the ethics commite (B.30.2.AKD.0.20.05.05).

Results: Both the transient tacyhpnea of the newborn group and the control group were consisted of 32 infants. Through the sequence display method, all the exons of the SCNN1A gene which codes the alpha subunit of epithelial sodium channels were displayed. Nevertheless, no significant change was found.

Conclusions: In conclusion, no relationship was established between the development of transient tacyhpnea of the newborn and the SCNN1A gene which is responsible for the synthesis of alpha subunit of epithelial sodium channels. However, this preliminary study is considered to be a threshold for an extended research with a wider range of patients to obtain more precise results. (Turk Arch Ped 2013; 48: 35-39)
\end{abstract}

Key words: Alpha subunit, epithelial sodium channel, genetic, SCNN1A gene, transient tachypnea of newborn

\section{Introduction}

Transient tachypnea of the newborn (TTN) is one of the causes of respiratory distress in term or near-term infants. Although its actual incidence is not known, it has been reported to occur in $0.33-0.5 \%$ of all births (1). It is thought that the disease mainly originates from the deficiency of maturation of the epithelial sodium channels $(\mathrm{ENaC})$ which have an important role in regulation of the movements of sodium and in absorption of the fluid in the alveoles $(2,3,4)$. However, the fact that the risk of asthma is increased in the advanced ages in patients with a diagnosis of TTN, different clinical pictures in each patient independent of risk factors and an unexplained familial tendency suggest that genetic factors may be involved in the background (5).
$\mathrm{ENaC}$ found in the epithelium of the airways is composed of three subunits including alpha, beta and gamma and the alpha subunit is accepted to be responsible of the functions of $\mathrm{ENaC}$ channel. As far as we know, the only study investigating the role of genetic variability related to TTN on the functions of $\mathrm{ENaC}$ belongs to Landman et al. (4). In this study, polymorphism in the SCNN1A gene which is responsible of the alpha subunit which is effective in the function of $\mathrm{ENaC}$ was investigated. However, the regions investigated were considerably limited and all the regions known should be studied to determine the role of any polymorphism which could explain the clinical variability.

The aim of this study was to investigate if any polymorphism related to the SCNN1A gene which is responsbile of the synthesis of the alpha subunit of $\mathrm{ENaC}$ has any role in development of TTN by viewing all the exons coding this gene. 


\section{Material and Method}

\section{Collection of the samples of the patient and control groups}

The newborns above the 37th gestational week followed up with a diagnosis of TTN in Akdeniz University, Medical Faculty Hospital, Neonatal Intensive Care Unit between July 2010 and January 2012 were included in this study.

The diagnosis of transient tachypnea of the newborn was made by postnatal clinical evaluation (a respiratory rate of $>60 /$ min in the first 24 hours, $>21 \%$ oxygen requirement) and marked lung graphy findings. Considering the clinical evaluation, lung graphy findings, blood values (complete blood count, CRP, sodium, potassium, calcium, glucose) and echocardiographic findings patients with pneumonia, pneumothorax, respiratory distress syndrome, early sepsis, congenital lung disease, congenital cardiac disease, left heart failure, eletrolyte disorders were excluded from the study. Infants of diabetic and preeclamptic mothers, infants of mothers with a diagnosis of asthma, infants with meconium aspiration syndrome and infants born with asphyxia were not included in the study.

Newborns who were born after the 37th gestational week, who did not have any health problem after delivery and who were followed up at the side of their mothers constituted the control group. Infants of mothers who had diabetes, asthma and preeclampsia during pregnancy were not included in the control group. Informed consent was obteined from the families in both groups and approval was obtained from the Akdeniz University Ethics Committee (B.30.2.AKD.0.20.05.05) for the study. The study was supported in the scope of Akdeniz University Scientific Research Project.

$2 \mathrm{cc}$ whole blood was taken into tubes with EDTA from both groups in the first five days of life and kept at $-80^{\circ}$. DNA isolation was performed in the department of Medical Biology and Genetics using Qiagene polyrase chain reaction (PCR) kit (HotStarTaq DNA Polymerase, 203205). Nanodrop spectrophotometer (Thermo scientific NanoDrop2000) was used to determine the quantity and quality of the DNAs obtained.

SPSS 18 was used for statistical analysis. Student's t-test was used for comparisons in terms of gestational week, weight, gender and mode of delivery between the study and control groups. Numeric data were expressed as mean (the least-the highest value) and a $p$ value of $<0.05$ was considered statistically significant.

\section{Primary design and polymerase chain reaction}

Genomic DNA (gDNA) base sequences related to the SCNN1A gene were obtained from the UCCS web site (http://genome.ucsc.edu/cgi-bin/hgGateway). In studies related to the SCNN1A gene, NM001159575 information was used. Polimerase chain reaction primers were designed with 50\& GC content using "Primer 3" program (Table 1). Agarose gel imaging system was used to view the obtained PCR products; $2 \%$ agarose gel was prepared, 2 ul PCR product was mixed with 10 ul bromphenol blue and loaded on the agarose gel. The samples were runned at $95 \mathrm{volt} / 30 \mathrm{~min}$. The residue excessive primers and dNTPs after PCR were inactivated using Exonuclease1 and
Shrimp alkaline phosphatase IT enzyme mixtures. One unit of exonuclease 1 and one unit of SAP were added to the PCR product. For the final products processed by Exo1-SAP, sequencing reaction was performed using BigDye Terminator v3.1(Cycle Sequencing Kit, 1009305M) and certain protocols.

Sequence cleaning reaction was performed with Qiagene Sequence cleaning columns (DyeEx 2.0 Spin Kit, 63204) using sefadex. The samples cleaned and prepared for sequence running reaction were transferred to optic plates.

The process of imaging was performed using $A B 3130$ (Applied Biosystems 3130 DNA sequencer) device. A certain running protocol was applied using Pop7 gel and $36 \mathrm{~cm}$ capillaries and the samples were runned. Mutation Surveyorv 4.0.5 trial version was used for analysis of sequence reactions. The numerical data related to the patients were expressed as the median (the least-the highest) values.

\section{Results}

45 patients with a diagnosis of TTN were evaluated in the study. 13 of the patients were excluded from the study because of various causes (four patients were infants of preeclamptic mothers, three patients were infants of diabetic mothers, two patients had abnormal renal function tests, three patients had minor cardiac pathology and one patient had pneumothorax). 32 patients who met the inclusion criteria and diagnosed as TTN had a birth weight of 3180 (2420-3950) g, a gestational age of 38.3 (37-41) weeks, a vaginal/cesarean delivery ratio of 7/25 and a male/female ratio of $24 / 8$. In the control group, the mean birth weight was found to be 3497 (2585-4200) g, the mean gestational age was found to be $39(38-41)$ weeks, the vaginal/cesarean delivery ratio was found to be $9 / 23$ and the male/female ratio was found to be 20/12. In the TTN group, the mean hospitalization time was found to be $6.03(2-13)$ days and the respiratory rate at presentation was found to be $77(65-120) / \mathrm{min}$. No statistically significant difference was found between the patients and control groups in terms of demographic properties. The general characteristics of the control and TTN groups are given in Table 2.

All exons coding the SCNN1A gene which is responsible of alpha $\mathrm{ENaC}$ were visualized with sequence imaging method; no difference was found between the exons of the patient and control groups.

\section{Discussion}

Although many studies have been conducted to explain the etiology and pathogenesis of transient tachypnea of the newborn, presence of potential genetic factors has not drawn adequate interest and adequate genetic studies have not been conducted. Studies investigating genetic polymorphism in patients with TTN are mostly related with surfactan protein-B and beta adrenergic receptor (ADRB 1-2) gene polymorphism $(6,7)$. In preterm infants, the relation of polymorphism occuring in the surfactan protein $B$ (SP-B) gene with respiratory distress syndrome (RDS) is well 
Table 1. Primers adjusted with the help of primer 3 program for the SCNN1A gene

\begin{tabular}{|l|l|c|c|}
\hline SCNN1A Primer & 5'--->3' & Tm ${ }^{\circ} \mathbf{C}$ & PCR product size \\
\hline SCNN1A_Exon 01-F & AGATAGCCCCAGAGGAGGAG & 61.4 & 230 \\
SCNN1A_Exon 01-R & TTCTTAAAGTGAAAGCCGGTG & 55.9 & 503 \\
\hline SCNN1A_Exon 02_1-F & GACAAAACTCGAAAGGTGGC & 57.3 & \\
SCNN1A_Exon 02_1-R & GGTAGGAGCGGTGGACTC & 61 & 460 \\
\hline SCNN1A_Exon 02_2-F & TCTAGCCCTCCACAGTCCAC & 61.4 & 407 \\
SCNN1A_Exon 02_2-R & CAGTGAGCACCTCAGCACC & 61 & \\
\hline SCNN1A_Exon 03-F & CAGACACTCGCTCCAGGG & 60.5 & 325 \\
SCNN1A_Exon 03-R & GGTCAGGAAAGGAGCGGAG & 61 & 236 \\
\hline SCNN1A_Exon 04-F & CACTCATTCTTGCCCTTGG & 57.3 & \\
SCNN1A_Exon 04-R & GACCCAGGGAAGCGGAC & 60 & 303 \\
\hline SCNN1A_Exon 05-F & ATTCCCTGCACCACCTACAC & 59.4 & \\
SCNN1A_Exon 05-R & AGGAGGTGAGCTCAAGGTAAG & 59.8 & 551 \\
\hline SCNN1A_Exon 06-F & GGCAAGGAAGGGAGAGTGG & 61 & \\
SCNN1A_Exon 06-R & GCCCCTCTGCAATCTGAG & 58.2 & 368 \\
\hline SCNN1A_Exon 07-08-F & AGAGAACCCAGAGGCACTTG & 59.4 & \\
SCNN1A_Exon 07-08-R & ACAAACAGGGGACTGAGAGG & 59.4 & \\
\hline SCNN1A_Exon 09-10-F & TGGGTGTGGGGTAGAGAAAG & 59.4 & \\
SCNN1A_Exon 09-10-R & CAGAGAAGGCCACAGCATTAC & 59.8 & \\
\hline SCNN1A_Exon 11-12-F & TTTGACACAACCCTATCCCTG & 57.9 & \\
SCNN1A_Exon 11-12-R & GCCCTGCTAAGTAAGACCCC & 61.4 & \\
\hline SCNN1A_Exon 13-F & TCTGCCAGAGTCCATCCAG & 58.8 & \\
SCNN1A_Exon 13-R & ATCCTTCAATCTTGCCAGGG & 57.3 & \\
\hline
\end{tabular}

Table 2. General characteristics of the patients with a diagnosis of TTN and the control group

\begin{tabular}{|l|c|c|c|}
\hline Özellikler & TTN group $\mathbf{n = 3 2}$ & Control group $\mathbf{n = 3 2}$ & $\mathbf{P}^{*}$ \\
\hline Male / Female & $24 / 8$ & $20 / 12$ & $>0.05$ \\
\hline Birth weight (g)a & $3180(2420-3950)$ & $3497(2585-4200)$ & $>0.05$ \\
\hline Gestational age (weeks)a $^{\mathrm{a}}$ & $38.3(37-40)$ & $39(38-41)$ & $>0.05$ \\
\hline Vaginal /Cesarean delivery & $7 / 25$ & $9 / 23$ & $>0.05$ \\
\hline
\end{tabular}

amedian (the least-the highest), ${ }^{*} p<0,05$ statistically significant

known (8,9). Tutdibi et al. (6) investigated the polymorphism which is involved in development of RDS and occurs in the previously defined SP-B gene in patients with a diagnosis of TTN and reported that no polymorphism was found in intron 4 and heterozygous 121 ins2 mutations in the SP-B gene. It is thought that there is a relation between endogeneous catecholamine level released during delivery and fluid clearance to provide fluid clearance in the lung. From this point of view, Aslan et al. (7) investigated the relation between ADRB1-2 receptor which is found in type II alveolar cells and activates NaKATPase by increasing $\mathrm{ENaC}$ expression and thus provides transepithelial sodium absorption with TTN. In this study, it was found that B1Gly49Gly genotype was significantly more prevalent in patients with a diganosis of TTN and presence of homozygous ADRB1 and Ser49Gly was a risk factor in these patients. In contrast, Satar et al. (10) investigated angiotensin converting enzyme (ACE) gene polymorphism in patients with a diagnosis of TTN and RDS and reported that no significant difference was found between ACE gene polymorphisms in the patient and control groups.

Genetic studies have associated some mutations which are found in different regions of exons with unknown function and outcomes with some diseases. The presence of mutations 
Table 3. Base changes and polymorphisms found in diseases related with SCNN1A

\begin{tabular}{|l|c|c|c|c|}
\hline Gene & Phenotype & Mutation & SNP & Reference \\
\hline SCNN1A & PHA $^{*}$ Type I & 1-BP DEL, 1449C & & Schaedel et al (12) \\
\hline SCNN1A & PHA* Type I & ARG 508 TER & & $\begin{array}{c}\text { Chang et al (13) } \\
\text { Bonny et al (14) }\end{array}$ \\
\hline SCNN1A & PHA $^{*}$ Type I & SER 562 LEU & & Schaedel et al (12) \\
\hline SCNN1A & PHA $^{*}$ Type I & 1-BP DEL, 729 A & & Schaedel et al (12) \\
\hline SCNN1A & PHA $^{*}$ Type I & 2-BP DEL, FS144TER & & Chang et al (13) \\
\hline SCNN1A & PHA $^{*}$ Type I & GLY 327 CYS & & Edelheit et al (15) \\
\hline SCNN1A & PHA $^{*}$ Type I & ARG 492 TER & & Bony et al (14) \\
\hline SCNN1A & Bronchiectasia & VAL 114 ILE & rs61759861 & Azad et al (16) \\
\hline SCNN1A & Bronchiectasia & TRP 493 ARG & rs5742912 & Azad et al (16) \\
\hline SCNN1A & Bronchiectasia & ARG 81 CYS & rs61759860 & Azad et al (16) \\
\hline
\end{tabular}

${ }^{*}$ Pseudohyperaldosteronism

FS, Frame Shift, TER: Terminal, DEL: Delesyon, SNP: single-nucleotide polymorphism

Amino acids: ARG: arginine, SER: serine, LEU: leucine, GLY: glycine, CYS: cycteine, VAL: valine, ILE: isoleucine, TRP: tryptophan.

associated with phenotype in regions the function of which can not be displayed with current methods, which are thought not to cause any change in the protein structure since they do not lead to a change in amino acids and which are even thought to have no significance on the gene is known. Advanced techniques are needed to determine the function of these mutations which appear to be insignificant or why they affect the phenotype. Similarly, it has been reported that polymorphisms called introns which are not normally found in the protein and which could not be determined in any way may be asociated with many diseases in unknown ways (11). All these associations can not be explained with the protein which is a gene product or with control of the gene. Therefore, one of the methods which can solve this problem seems to find which isoform of the SCNN1A gene which is the searched gene for the disease we are investigating is synthesized and which protein is produced and if these proteins fulfill their potential functions and to investigate their novel functions, if present.

In the single study which investigated the genetic role of $\mathrm{ENaC}$ in TTN, Landman et al. (4) investigated the polymorphisms in exon 12th and 13th regions which synthesize the parts of alpha $\mathrm{ENaC}$ (SCNN1A) passing the cellular membrane in newborns with TTN, but could not show an association. In our study, imaging by sequaencing method was performed in the patient and control groups to determine potential base changes in exon structures which constitute protein coding parts of the $\mathrm{ENaC}$ gene. In contrast to Landman et al. (4) all exons coding the ENaC (SCNN1A) gene were examined with this method, but no significant association could be found.

Base changes or polymorphisms which the researchers found to be associated with SCNN1A and which are associated with diseases are shown in Table 3. In both diseases in which polymorphisms were found (bronchiectasia and peudohyperaldosteronism), it was thought that respiratory problems were related with loss of function of ENaC. However, no relation was found with the polymorphisms found in these two diseases and TTN in our study.

Maintenance of a high number of patients in studies is the most important factor which increases the reliability of the results and which provides determination of gene mutations which have little action in terms of clinical findings. In addition, screening the whole gene instead of only the regions which are known to be significant in terms of function seems to be a more beneficial approach in obtaining novel and original results. Therefore, it was preferred to screen the whole gene instead of the candidate region which was thought to be related with the disease in our study, since there was no information about the genetic background responsible of the phenotype in terms of TTN. However, we think that the low number of patients who we could include in our study might have decreased the possibility of obtaining positive data in this large screening.

At the end of our study, all exons coding the SCNN1A gene were visualized with sequence imaging method in our patients with TTN, but no change could be found. Therefore, we think that planning a new study which can screen the whole genome including a larger number of patients and detailed examination of the functions of the gene in living creatures with knock-out or knock-down methods in transgenic animal models would be beneficial in terms of better evaluation of the genetic aspect of the disease.

\section{Conflict of interest: None declared.}




\section{References}

1. Hansen AK, Wisborg K, Uldbjerg N, Henriksen TB. Risk of respiratory morbidity in term infants delivered by elective caesarean section: cohort study. BMJ 2008; 336(7635): 85-87.

2. Shehata MF Regulation of the epithelial sodium channel $[\mathrm{ENaC}]$ in kidneys of salt-sensitive Dahl rats: insights on alternative splicing. Int Arch Med 2009; 2(1): 28.

3. Helve $\mathrm{O}$, Pitkänen $\mathrm{O}$, Janér $\mathrm{C}$, Andersson $\mathrm{S}$. Pulmonary fluid balance in the human newborn infant. Neonatology 2009; 95(4): 347-352.

4. Landmann E, Schmidtpott M, Tutdibi E, Gortner L. Is transient tachypnoea of the newborn associated with polymorphisms in the epithelial sodium channel encoding gene? Investigation of the second transmembrane spanning domain of the alpha subunit. Acta Paediatr 2005; 94(3): 317-323.

5. Guala A, Carrera P, Pastore G, Somaschini M, Ancora G, Faldella G, De Filippi P, Ferrero F, Guarino R, Danesino C. Familial clustering of unexplained transient respiratory distress in 12 newborns from three unrelated families suggests an autosomal-recessive inheritance. Scientific World Journal 2007; 7: 1611-1616.

6. Tutdibi E, Hospes B, Landmann E, Gortner L, Satar M, Yurdakök M, Dellagrammaticas H, Ors R, llikkan B, Ovali F, Sarman G, Kumral A, Arslanoglu S, Koc H, Yildiran A. Transient tachypnea of the newborn (TTN): a role for polymorphisms of surfactant protein B (SP-B) encoding gene? Klin Padiatr 2003; 215(5): 248-252.

7. Aslan E, Tutdibi E, Martens S, Han Y, Monz D, Gortner L. Transient tachypnea of the newborn (TTN): a role for polymorphisms in the betaadrenergic receptor (ADRB) encoding genes? Acta Paediatr 2008; 97(10): 1346-1350.

8. Floros J, Veletza SV, Kotikalapudi P, Krizkova L, Karinch AM, Friedman C, Buchter S, Marks K. Dinucleotide repeats in the human surfactant protein-B gene and respiratory-distress syndrome. Biochem J 1995: 305: 583-590.

9. Makri V, Hospes B, Stoll-Becker S, Borkhardt A, Gortner L. Polymorphisms of surfactant protein $B$ encoding gene: modifiers of the course of neonatal respiratory distress syndrome? Eur J Pediatr 2002; 161(11): 604-608.
10. Satar M, Tașkın E, Özlü F, Tuli A, Özcan K, Yıldızdaş HY. Polymorphism of the angiotensin-converting enzyme gene and angiotensin-converting enzyme activity in transient tachypnea of neonate and respiratory distress syndrome. J Matern Fetal Neonatal Med 2012: 25(9): 17121715.

11. Pfeufer A, Jalilzadeh S, Perz S. Common variants in myocardial ion channel genes modify the QT interval in the general population: results from the KORA study. Circ Res 2005: 96(6); 693-701.

12. Schaedel C, Marthinsen L, Kristoffersson AC, Kornfält R, Nilsson KO, Orlenius B, Holmberg L. Lung symptoms in pseudohypoaldosteronism type 1 are associated with deficiency of the alpha-subunit of the epithelial sodium channel. J Pediatr 1999; 135(6): 739-745.

13. Chang SS, Grunder S, Hanukoglu A, Rösler A, Mathew PM, Hanukoglu I, Schild L, Lu Y, Shimkets RA, Nelson-Williams C, Rossier BC, Lifton RP. Mutations in subunits of the epithelial sodium channel cause salt wasting with hyperkalaemic acidosis, pseudohypoaldosteronism type 1. Nature Genet 1996; 12(3): 248-253.

14. Bonny O, Chraibi A, Loffing J, Jaeger NF, Gründer S, Horisberger JD, Rossier BC. Functional expression of a pseudohypoaldosteronism type I mutated epithelial $\mathrm{Na}+$ channel lacking the pore-forming region of its alpha subunit. J Clin Invest 1999; 104(7): 967-974.

15. Edelheit O, Hanukoglu I, Gizewska M, Kandemir N, TenenbaumRakover Y, Yurdakök M, Zajaczek S, Hanukoglu A. Novel mutations in epithelial sodium channel (ENaC) subunit genes and phenotypic expression of multisystem pseudohypoaldosteronism. Clin Endocrinol (Oxf) 2005; 62(5): 547-553.

16. Azad AK, Rauh R, Vermeulen F, Jaspers M, Korbmacher J, Boissier B, Bassinet L, Fichou Y, des Georges M, Stanke F, De Boeck K, Dupont L, Balascáková M, Hjelte L, Lebecque P, Radojkovic D, Castellani C, Schwartz M, Stuhrmann M, Schwarz M, Skalicka V, de Monestrol I, Girodon E, Férec C, Claustres M, Tümmler B, Cassiman JJ, Korbmacher $\mathrm{C}$, Cuppens $\mathrm{H}$. Mutations in the amiloride-sensitive epithelial sodium channel in patients with cystic fibrosis-like disease. Hum Mutat 2009; 30(7): 1093-103.

17. Mekus F, Ballmann M, Bronsveld I, Dörk T, Bijman J, Tümmler B, Veeze HJ. Cystic-fibrosis-like disease unrelated to the cystic fibrosis transmembrane conductance regulator. Hum Genet 1998; 102(5): 582-586. 